\title{
Tratamiento informativo y cobertura electoral en Querétaro: Una mirada a la elección estatal de 2015
}

\author{
Informative treatment and electoral coverage in Querétaro: A look at the \\ 2015 state election
}

\author{
Gabriel A. CORRAL VELÁZQUEZ \\ Universidad Autónoma de Querétaro \\ corral@uaq.mx
}

Recibido: 30 de junio del 2017

Aceptado: 22 de septiembre 2017

\begin{abstract}
Resumen
El estado de Querétaro cuenta con tres alternancias electorales (1997, 2009, 2015) Lo cual podría mostrar al exterior una pluralidad política y una madurez electoral. A partir de lo expuesto se puede sostener que en la prensa y, en los espacios de deliberación, prevalecen prácticas políticas contradictorias muestran continuidades propias del régimen político autoritario. Más allá de los nuevos marcos regulatorios en los medios electrónicos, en la prensa diaria continúan los convenios y los acuerdos publicitarios enfatizados en los procesos electorales.

El presente trabajo tiene el propósito de mostrar un acercamiento a la cobertura informativa durante el proceso electoral del año 2015 en el estado de Querétaro. A partir de los discursos presentados en la prensa diaria de la capital del estado, se pretende poner en perspectiva cómo se configura la esfera pública en un contexto de alternancia electoral.
\end{abstract}

\section{Abstract}

The state of Querétaro has three electoral alternations (1997, 2009, 2015) which could show abroad a political plurality and electoral maturity. Based on the above, it can be argued that contradictory political practices prevail in the press and, in spaces for deliberation, show continuities typical of the authoritarian political regime. Beyond the new regulatory frameworks in electronic media, in the daily press the agreements and advertising agreements emphasized in electoral processes continue.

The present work has the purpose of showing an approach to the informative coverage during the electoral process of 2015 in the state of Querétaro. Based on the speeches presented in 
the daily press of the state capital, it is intended to put into perspective how the public sphere is configured in a context of electoral alternation.

Palabras Clave: Alternancia Electoral; Esfera Pública; Discurso Key Words: Electoral Alternation; Public Sphere; Speech.

\section{Introducción}

Hoy en día la esfera pública representa el espacio en donde la acción de los actores sociales disputa la visibilidad, la influencia en los procesos de constitución de la agenda pública y de las decisiones colectivas. En la esfera pública se manifiesta la fuerza o debilidad de los actores, la capacidad de los ciudadanos para interactuar y participar en la definición de los asuntos públicos.

Se pretende poner en perspectiva por medio de analizar la configuración de la esfera pública la visibilidad de los actores sociales y las prácticas que dan origen a la cultura y participación política en Querétaro en el contexto del proceso de democratización. Durante este proceso se han generado tensiones entre quienes buscan expresión y visibilidad en la esfera pública y actores que pretenden mantener los asideros culturales que han dominado los espacios de deliberación pública. La ruta que sigue este ensayo es la siguiente: se presenta una síntesis de las relaciones históricas entre las élites, el proceso de democratización, los espacios de interacción pública y la permanencia de las prácticas políticas a partir de ejemplos recuperados de la prensa local.

\section{Acercamiento Conceptual}

En los últimos años hemos asistido a una gran cantidad de cambios y apropiaciones diversas de referentes discursivos durante los procesos electorales. Actores sociales, políticos y ciudadanía en general han adoptado discursos democráticos en forma masiva (tal como sucede en México desde la década de 1980) En este sentido, es lógico preguntarse qué correlación existe entre representaciones, discursos y prácticas cotidianas en materia política. Si por décadas el discurso de la Revolución Mexicana fue cosificado hasta 
convertirlo en un espectro sin vida ni realidad, lo mismo puede decirse del vendaval de expresiones democratizantes que la clase política y los medios de comunicación han incorporado a sus discursos diarios.

La brecha entre promesas y realidad sigue inmutable en la rutina sexenal: desde aquel lema de Democracia y Justicia Social que usara Miguel Alemán en su campaña presidencial de 1946, pasando por la Renovación Moral de Miguel de la Madrid en 1982 o el Cambio prometido por Vicente Fox Quezada en las elecciones del año 2000; se puede decir no tan solo que la gran cantidad de términos relacionados con el tema es norma en México sino que la configuración de las sociedades verdaderamente democráticas no se produce a base de dogmatismo conceptual sino de pruebas reales. En ello, la cobertura electoral y el tratamiento informativo durante los procesos electorales ha sido pieza fundamental en la difusión de dichos contenidos y en la influencia que éstos han tenido en la toma de decisiones al momento de elegir a un candidato u otro, al menos desde 1996.

La posibilidad de la interacción y la comunicación no coactiva entre actores sociales es la base de la idea democracia liberal, no es ocioso preguntarse si en Querétaro o en cualquier otro lugar del país, los factores reales del poder -clase empresarial y administración públicaabrieron los canales de comunicación con la sociedad civil para que las expresiones de inconformidad, proposición o transparencia tuvieran su lugar en el proceso de toma de decisiones que afecta al desarrollo social, económico y cultural.

Es en este sentido que se sostiene la idea de comprender la esfera pública como una idealización de procesos históricos. Conviene precisar, en este sentido, que es en ella donde tienen lugar las prácticas a las que se hace referencia y en donde se toman las decisiones en torno a lo político. Esta definición aplica para todos aquellos lugares donde la participación política se establece por medio del diálogo. Diálogo basado en el examen libre y racional de los asuntos comunes mediante una interacción discursiva entre actores sociales. Este es un escenario intermedio entre el Estado y el mercado donde se produce y circula el 
pensamiento crítico en el cual se puede incluir: Discurso, debate y deliberación. Fundamentalmente en los procesos electorales cobra sentido esta conceptualización, puesto que es ellos cuando se pone de manifiesto la deliberación pública y la toma de decisiones.

La esfera pública planteada por Habermas (1981) y sus efectos duraderos en el debate cultural sostienen la idea de un espacio donde la libre asociación entre ciudadanos y la acción discursiva construyen una poderosa opinión pública fuerte y soberana frente al Estado y el mercado capturó la imaginación no sólo de la intelectualidad crítica sino incluso de las clases medias y los movimientos sociales. Un mundo basado en una pluralidad de perspectivas, fundado sobre un diálogo inclusivo y orientado hacia el consenso en medio de una libertad comunicativa que solo puede nacer de unas expectativas de cooperación entre personas reflexivas que asumen un proyecto vital en conjunto (Habermas, 1981).

El estado de Querétaro se ha caracterizado por ser una de las entidades con mayor índice de participación en los diferentes procesos electorales. Concretamente en el de 2015, de acuerdo con datos del INE, el $57 \%$ de la lista nominal de electores se presentó a las urnas, ocupando el quinto lugar en participación electoral siendo Yucatán el primer lugar, con un 68.2 por ciento, seguido de Nuevo León con un 58.5 por ciento. ${ }^{\text {. }}$

Quizá los medios poco tengan que ver con este índice, sin embargo, es innegable el papel que tienen en la configuración de espacios de deliberación y la toma de decisiones por parte de la ciudadanía. En este sentido, la agenda de temas y la discusión de los mismos forman el engranaje que en la esfera pública cobra sentido en un contexto de democratización. Al mismo tiempo se relaciona con la cultura política y la capacidad de la ciudadanía para movilizarse durante los procesos electorales. Al final los medios de comunicación con el establecimiento de agendas mediáticas y su influencia sobre la ciudadanía configuran parte del mapa del mundo que sigue marcando nuestras vidas, nuestras percepciones y también nuestras decisiones.

\footnotetext{
${ }^{1}$ Datos de participación ciudadana del INE publicados en junio de 2015 consultado: http://adninformativo.mx/ocupa-queretaro-5to-lugar-en-participacion-ciudadana-durante-elecciones/
} 


\section{Cobertura Informativa y esfera pública en Querétaro}

En el análisis que se realizó durante el proceso electoral se pretendió observar el tratamiento informativo y el propio discurso de los candidatos de los partidos políticos con mayor representación en la entidad.

La esfera pública, en este sentido, posee una cantidad importante de temas y asuntos de interés general. Lo publicado en la prensa corresponde a una parte representativa. Es ahí donde cabe el planteamiento de Habermas (1981) al referir que los medios no son la esfera pública, sino que representan una parte de ella. De esta forma, lo que se analiza es la puesta en común, a partir de las notas establecidas como discurso. Por tal motivo se ha decido establecer una agenda de temas concretos que refieran a estos aspectos en el discurso periodístico analizado en el contenido de las noticias.

En estos temas se puede anotar cómo los candidatos al gobierno del estado plantean su agenda y los propios medios utilizan la misma como anclaje para la publicación y jerarquización informativa; construyendo de esta manera un significado de ejercicio de gobierno o con valores que establezca alguna idea para englobar en el proceso de democratización y en la propia inclusión de actores en la esfera pública.

Desglose por temas.

a) Desarrollo de Infraestructura. En este tema se eligieron todas las notas en donde se habla de desarrollo urbano, vivienda, caminos, obra pública. No solamente que los actores políticos refirieran discursivamente sobre el tema; sino también otros actores de la sociedad civil expusieron demandas respecto de la necesidad de obras.

b) Participación Social. Aquí se eligieron las noticias que hablaran principalmente de la sociedad civil o que invitaran a la toma de decisiones. Sectores económicos y académicos tomaron postura en este ámbito. 
c) Manejo de los Recursos Públicos. Fue tema de debate durante el proceso electoral y en las semanas posteriores. La ley 3 de 3 y el gasto de los partidos políticos durante las elecciones que tuvieron lugar en junio fueron parte de los asuntos que abarcaron la discusión sobre este tema.

Con estos tres temas se puede sostener la idea de que la práctica del discurso en la prensa implica transformaciones de textos originales, una conferencia de prensa, una entrevista, un mitin; en un artículo. El texto es probable que haya pasado por una serie de versiones y revisiones. En este sentido, es un discurso reproducido ya que se transformó en una cadena de acontecimientos vinculados a un proceso institucional. La práctica del discurso periodístico, bajo esta idea, es compleja, ya que articula características del discurso de la fuente con las del discurso "objetivo" de la prensa y el consumo del mismo por parte de la ciudadanía.

Para analizar la cobertura informativa, el discurso que en la prensa reproduce se utilizó una guía propuesta por Torfing(1999), la cual recupera elementos de diversos enfoques analíticos del discurso.

De esta propuesta se recuperan los siguientes puntos para trabajar con las noticias:

1) Punto nodal. Hace referencia a la agrupación de varios elementos discursivos bajo un significado o punto de referencia. (Torfing, 1999).

2) Articulación. Los discursos se convierten de esta manera en instrumentos para el ejercicio del poder. "El éxito de la articulación nunca es total, debido al carácter abierto de la vida social, consecuencia de la infinita creatividad interpretativa de los seres humanos" (Torifing, 1999).

3) Antagonismo. Hace referencia al proceso en el que los actores se expresan su intento por alcanzar posiciones de poder y en la formación de antagonismos sociales. A partir de esto se construyen los oponentes. (Torfing, 1999). 
4) Lógica de equivalencia. El punto nodal es el eje que articula la lógica de equivalencia. Esto es establecer los rasgos específicos de cada discurso en torno a la forma discursiva más simple sobre el cual pretende la adscripción de un mayor número de sectores sociales y, de este modo, convertirse en una herramienta política más (Torfing, 1999).

5) Posiciones y subjetividad política. Refiere a las diferentes posiciones sobre las que una persona se identifica en relación a un determinado asunto de interés colectivo referido en el discurso a partir de las distintas formas de actuación por medio de las cuales se participa en la sociedad. (Torfing, 1999).

En la figura se puede observar la manera en cómo cada uno de los elementos se relacionan en torno al punto nodal y cómo se consolida los diversos discursos en la esfera pública.

Figura 1 Esquema de análisis

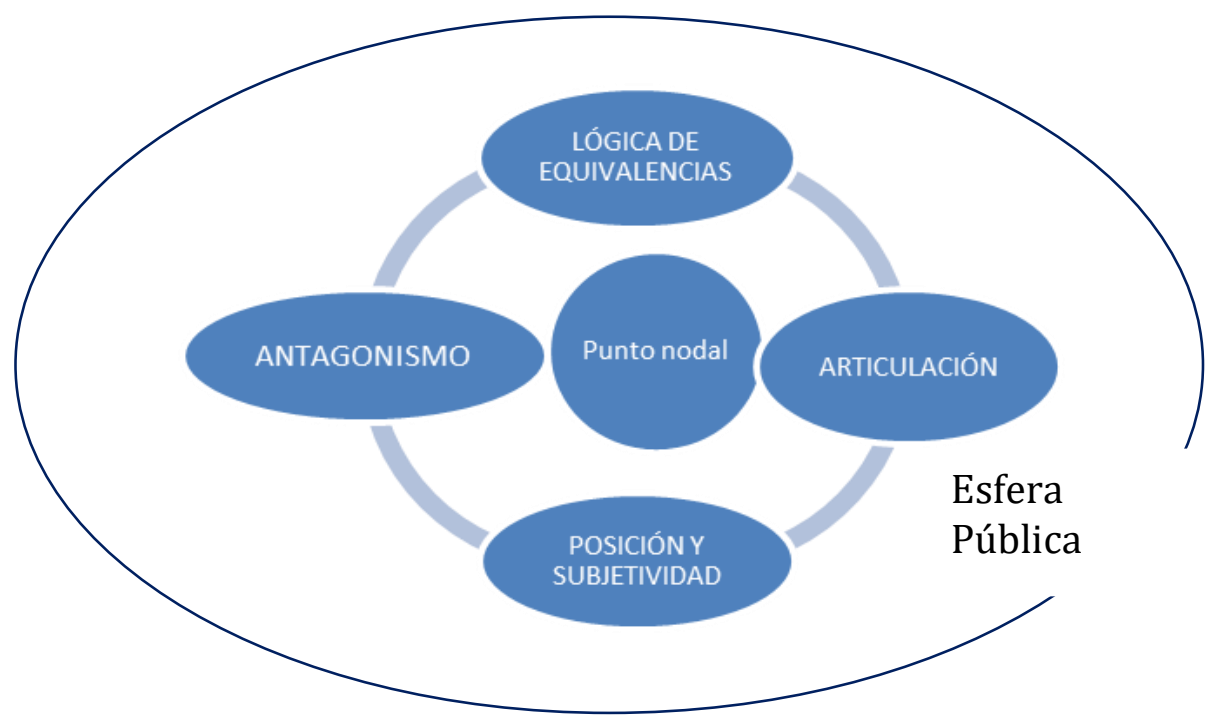

Teniendo en cuenta estos elementos, se procedió a la tarea de recuperar el corpus de análisis de la siguiente forma. 


\section{Producción Informativa en Querétaro}

La producción informativa de los informantes y de los medios en que laboran es muy diversa. El periódico $A M$ publica en promedio a la semana en temas relacionados con política y asuntos públicos un promedio de 25 a 30 notas y alrededor de 3 a 5 entrevistas dependiendo de los temas semanales.

El periódico Noticias publica en promedio 48 notas informativas sobre temas que tiene relación con política o asuntos de interés público y 4 entrevistas.

Diario de Querétaro publica a la semana un promedio de 50 notas informativas relacionadas con los temas de interés.

El periódico Capital Querétaro tiene una particularidad, este diario sólo publica de lunes a viernes, por tal motivo las notas publicadas son menos que los diarios que circulan siete días a la semana. Este periódico publica en promedio 17 notas. Es el diario en circulación que menos información pública.

Tabla 1

Producción Informativa en Querétaro

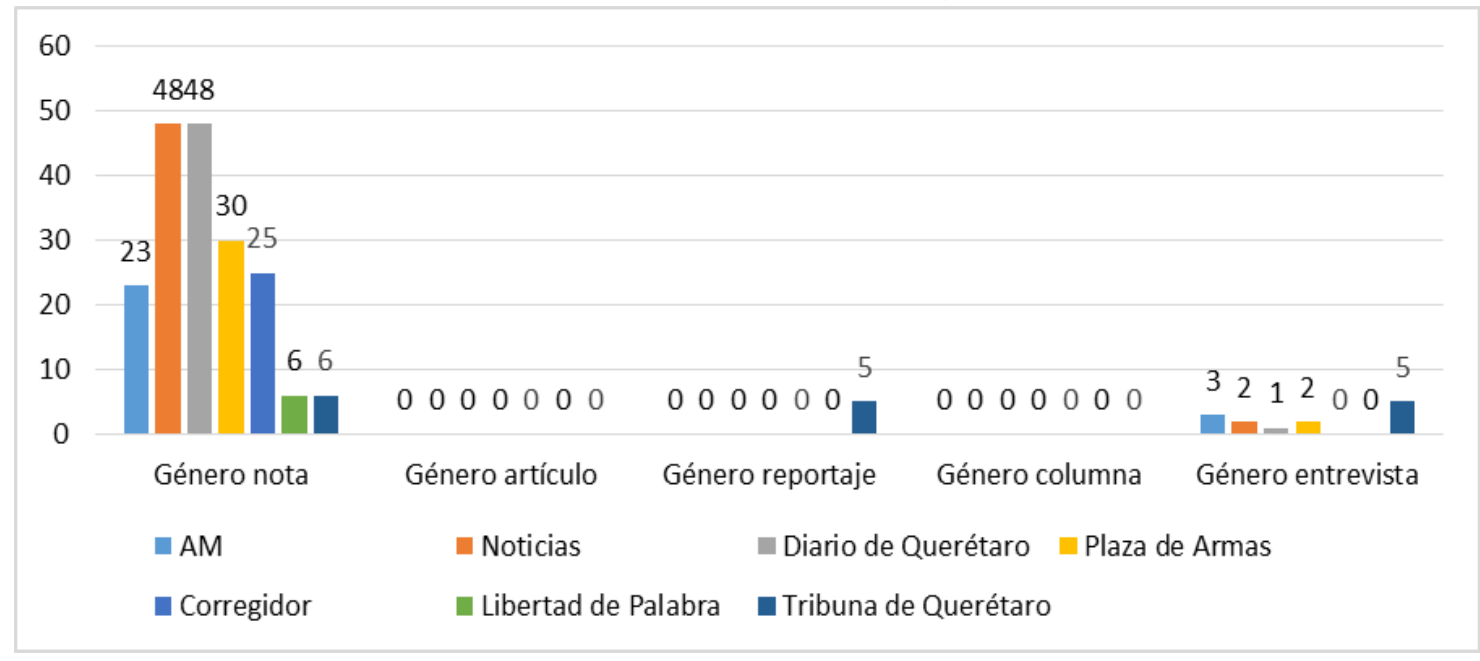

Fuente: Elaboración Propia

Facultad de Ciencias Sociales y de la Comunicación Universidad de La Laguna 
Se puede mostrar en este mapa de producción informativa que los géneros de opinión como la columna, artículo de fondo, crónica o editorial, no forman parte de la práctica cotidiana en la producción informativa de la prensa escrita de la ciudad de Querétaro.

Sólo en los semanarios Tribuna de Querétaro y Libertad en palabra, existen reportajes o entrevistas relacionadas con temas distintos a los de los diarios ${ }^{2}$.

Con este mapa se estableció la agenda de análisis respecto a la propia producción de noticias. En este sentido se establecieron los criterios argumentales y prácticos para el material informativo que se está trabajando y el lugar que ocupan dentro de la agenda de la propia prensa los temas de interés político.

En las noticias, quiénes son los actores que producen el discurso y cuáles son las estrategias que utilizan, para a partir de las temáticas seleccionadas. Al mismo tiempo se puede dar cuenta de cómo los distintos actores que intervienen en la producción informativa de la prensa pretenden influir para dominar en el debate y por ende en la esfera pública, finalmente buscan la legitimación respecto de grupos influyentes, como son las élites económicas o políticas.

Con este mapa de producción informativa se realizó la selección de notas de la siguiente manera.

\footnotetext{
${ }^{2}$ En el análisis no fueron incluidos dichos semanarios debido a que no son publicaciones diarias y el estudio fue hecho sobre el género noticia publicada en rotativos de circulación diaria.
} 
Tabla 2

Número de noticias para el análisis

\begin{tabular}{|c|c|}
\hline Periódico & Número de noticias \\
\hline Diario de Querétaro & 80 \\
\hline Noticias & 80 \\
\hline AM & 60 \\
\hline Capital & 35 \\
\hline El Universal Querétaro & 50 \\
\hline Plaza de Armas & 45 \\
\hline Total & \\
\hline
\end{tabular}

Fuente: Elaboración Propia

Por tema se ha dividido de la siguiente manera. El periódico que más información presenta es Diario de Querétaro y el tema que más ocupó espacio durante el proceso electoral fue la iniciativa 3 de 3 y la discusión entre los candidatos sobre presentar o no tal declaración.

Con este corpus se trabajó el análisis. El proceso de selección se dio a partir de los temas y el contenido de la información, en este sentido se cuidó que en el contenido hubiera elementos que tuvieran un margen de interpretación de acuerdo al esquema de análisis.

En la mayoría de las notas que conforman este corpus se encuentra rasgos en común; la mayoría de ellas reproduce declaraciones o conferencias de prensa. Durante el proceso electoral se agrega otro escenario que son los mítines de campaña. En la parte que corresponde al tiempo poselectoral regresan las declaraciones o las conferencias de prensa.

Este aspecto es destacado porque marca el contexto en el que el discurso es construido. No es lo mismo citar una declaración, discurso producido específicamente a pregunta de un periodista, a recuperar un mensaje en un mitin de campaña, el cual está producido para un auditorio más amplio de electores al que se busca convencer. Si bien ambos son "empaquetados" en el género nota informativa (que es lo que se está analizó) el contexto de origen permite ubicar bajo qué circunstancias se están haciendo los señalamientos por parte 
del actor, al momento de desglosar los contenidos de la nota y de esta manera revisar el tratamiento informativo de la prensa.

Con estos elementos se procedió a realizar la recuperación de los datos empíricos para el análisis.

\section{Ubicación de las notas en el cuerpo de los periódicos}

Las notas que se seleccionaron están ubicadas en las secciones de información local. En los cuatro periódicos estas secciones obedecen a la primera plana y sus interiores. Salvo el caso de noticias, que, a pesar de ser el único diario de capital local, casi todas sus notas de ocho columnas o son notas nacionales o son notas de policía y también no todas locales. En los otros periódicos sus notas de ocho columnas son locales.

En la selección establecida, se revisaron las noticias y en la gran mayoría son notas que se encuentran en las páginas interiores. Las notas de ocho columnas durante los meses previos a la campaña electoral eran en su mayoría noticias relacionadas con el gobierno del estado. No necesariamente del gobernador (José Calzada Rovirosa 2009 - 2015) quien se caracterizó por ser un gobernador presente incluso durante las campañas electorales, al mismo tiempo se le consideró un gobernador bien evaluado y aceptado por la ciudadanía.

Durante la campaña electoral fue combinada la publicación de notas en ocho columnas. Aparecían los candidatos. En gran mayoría el candidato del Partido Acción Nacional y en otras ocasiones el candidato del PRI. Una vez terminado el proceso electoral, las notas de ocho columnas fueron para el candidato triunfador, en calidad de gobernador electo y para las noticias que surgían entorno al cambio de gobierno.

Las notas de interiores, son las que ofrecen un panorama más amplio. En ellas aparecen más actores. Desde partidos políticos hasta actores de la sociedad civil. Los temas suelen ser más variados, pero siempre relacionados con cuestiones políticas. 
Aunque no es igual una nota de interiores que una de primera plana, la intencionalidad de los discursos y de la producción varía en ese sentido. Para el análisis se utilizó el mismo esquema fueran notas que provinieran de una u otra parte del periódico.

\section{Actores principales de las notas}

Una vez realizada la selección de las notas se realizó un conteo para ubicar los actores. En el periodo seleccionado, esto se realizó para ubicar quienes son los actores que reciben más cobertura y establecer una primera aproximación a quienes dominan los espacios noticiosos en la prensa local.

Para ello se dividió por meses la siguiente tabla en el espacio menciones se contabilizó las veces que aparecía el actor y en espacio, si era interiores o en primera plana. La cobertura al gobierno (estatal o municipal) y la legislatura decrecieron considerablemente en los meses de mayo y junio en tanto que la información relacionada con partidos políticos y organismos públicos autónomos creció en este periodo. 
Tabla 3

Cobertura en prensa escrita

\begin{tabular}{|c|c|c|c|c|c|c|}
\hline \multirow[b]{2}{*}{ Actor } & \multicolumn{2}{|c|}{31 de marzo/ 18 abril } & \multicolumn{2}{|r|}{21 abril /16 mayo } & \multicolumn{2}{|c|}{19 mayo /6 junio } \\
\hline & M & Espacio & M & Espacio & M & Espacio \\
\hline Partido Político & 25 & Interiores & 30 & $\begin{array}{ll}\text { Primera } & \text { plana/ } \\
\text { Interiores } & \end{array}$ & 30 & $\begin{array}{l}\text { Primera plana/ } \\
\text { Interiores }\end{array}$ \\
\hline $\begin{array}{l}\text { Gobierno } \\
\text { Estatal }\end{array}$ & 30 & Primera plana & 25 & $\begin{array}{l}\text { Primera plana/ } \\
\text { Interiores }\end{array}$ & 20 & $\begin{array}{l}\text { Primera plana/ } \\
\text { Interiores }\end{array}$ \\
\hline $\begin{array}{l}\text { Gobierno } \\
\text { Municipal }\end{array}$ & 30 & $\begin{array}{l}\text { Primera } \\
\text { plana/Interiore } \\
\mathrm{s}\end{array}$ & 20 & & 10 & \\
\hline Legislatura & 24 & Interiores & 20 & Interiores & 15 & Interiores \\
\hline $\begin{array}{l}\text { Instituciones } \\
\text { públicas } \\
\text { autónomas }\end{array}$ & 15 & Interiores & 20 & Interiores & 20 & Interiores \\
\hline $\begin{array}{l}\text { Organismos de } \\
\text { la sociedad civil }\end{array}$ & 15 & Interiores & 15 & Interiores & 15 & Interiores \\
\hline $\begin{array}{l}\text { Ciudadanos no } \\
\text { identificados } \\
\text { con algún } \\
\text { partido /ONG }\end{array}$ & 10 & Interiores & 10 & Interiores & 10 & Interiores \\
\hline
\end{tabular}

M: Número de Menciones

Fuente: Elaboración propia

El conteo es un promedio de menciones en función de las notas publicadas. De acuerdo a como se realizó la selección de las notas aparece una nota diaria de los actores gubernamentales, en tanto que de organismos de la sociedad civil y ciudadanos no identificados con partidos o con ONG son realmente escasas. Esto puede dar una idea de cómo está desequilibrada la cobertura de noticias. $Y$ ayuda para sostener el argumento de que los medios, en este caso la prensa escrita de la ciudad de Querétaro, solo cubre una parte de los debates que se generan en la esfera pública. 
Al ser un conocer a qué candidato se le otorgó mayor espacio en la prensa de la ciudad de Querétaro, se realizó el conteo relacionado con este tema, solo para tener un dato extra entorno a quién se reunía la mayor cantidad de espacios noticiosos.

Tabla 4

Cobertura candidatos a gobernador del estado

\begin{tabular}{|l|l|l|l|l|l|l|}
\hline & $31 \mathrm{marzo} /$ & \multicolumn{1}{c|}{18 abril } & \multicolumn{2}{c|}{21 abril / } & \multicolumn{1}{c|}{16 mayo } & 19 mayo / 6 \\
\hline Candidato & Menciones & Espacio & Menciones & Espacio & Menciones & Espacio \\
\hline $\begin{array}{l}\text { PRI. } \\
\text { Roberto } \\
\text { Loyola Vera }\end{array}$ & 35 & $\begin{array}{l}\text { Primera } \\
\text { Plana } \\
\text { Interiores }\end{array}$ & 30 & $\begin{array}{l}\text { Interiores/ } \\
\text { Primera } \\
\text { plana }\end{array}$ & 30 & $\begin{array}{l}\text { Interiores/ } \\
\text { Primera } \\
\text { plana }\end{array}$ \\
\hline $\begin{array}{l}\text { PAN. } \\
\text { Francisco } \\
\text { Domínguez } \\
\text { Servién }\end{array}$ & 30 & $\begin{array}{l}\text { Interiores/ } \\
\text { Primera } \\
\text { plana }\end{array}$ & 30 & $\begin{array}{l}\text { Interiores/ } \\
\text { Primera } \\
\text { plana }\end{array}$ & 30 & $\begin{array}{l}\text { Interiores/ } \\
\text { Primera } \\
\text { plana }\end{array}$ \\
\hline $\begin{array}{l}\text { PRD. } \\
\text { Adolfo } \\
\text { Camacho } \\
\text { Esquivel }\end{array}$ & 10 & $\begin{array}{l}\text { Primera } \\
\text { Plana/Inte } \\
\text { riores }\end{array}$ & 20 & Interiores & 20 & Interiores \\
\hline
\end{tabular}

Fuente: Elaboración Propia

Los candidatos del PRI y el PAN recibieron todos los días cobertura de sus campañas. Salvo el caso del candidato del PRD y de otros partidos pequeños. Sin ser una cobertura equilibrada, la prensa cumplió con la publicación de notas alusivas a la campaña electoral de todos los partidos políticos. Esto de acuerdo a la legislación vigente durante los periodos electorales.

Este conteo permitió ubicar a los actores que más aparecen en las notas y tener un mapa más claro de la cobertura que tienen los distintos actores sociales. Quedó claro a partir de este promedio que los medios cubren más las fuentes gubernamentales que a otros actores de la esfera pública. Los candidatos del PRI y el PAN recibieron todos los días cobertura de sus campañas. Salvo el caso del candidato del PRD y de otros partidos pequeños. Sin ser una cobertura equilibrada, la prensa cumplió con la publicación de notas alusivas a la 
campaña electoral de todos los partidos políticos; sin embargo, fue evidente el sesgo informativo hacia el candidato del PRI, Roberto Loyola Vera.

\section{Acercamiento al análisis de la cobertura noticiosa}

A partir de los puntos que se revisaron se hizo el desglose del análisis de la siguiente manera.

Articulación. Por el contexto en el que se desarrolló el trabajo el tema del uso de los recursos públicos apareció de manera constante por la iniciativa 3 de 3 que puso en el debate el candidato Domínguez Servién del Partido Acción Nacional. Cobró importancia debido a que el mayor cuestionamiento se hizo debido al uso del dinero en las campañas electorales y los postulados que establece esta iniciativa. o a los salarios de los funcionarios. La participación social y el desarrollo de la infraestructura fueron los temas de articulación en menor medida.

En todos los casos hubo alusiones a la transparencia y a la contienda democrática. Ejemplo de ello son las siguientes frases:

- "Voy a ganar con 8 puntos: Pancho" FDS ${ }^{3}(9 / 04 / 15)$

- "El proceso es democrático, pues hay varios contendientes, es así como se demuestra la democracia, cada quien con sus ideas y proyectos:" Loyola RLV ${ }^{4}(14 / 04 / 15)$

- "Dispuesto a entregar 3 de 3: Pancho" FDS (24/04/15)

- Soy un libro abierto: Loyola RLV (24/04/15)

\footnotetext{
${ }^{3}$ FDS: Iniciales de Francisco Domínguez Servién. Candidato al gobierno del estado de Querétaro por el Partido Acción Nacional.

${ }^{4}$ RVL: Iniciales de Roberto Loyola Vera. Candidato al gobierno del estado de Querétaro por el Partido Revolucionario Institucional
} 
En esta primera etapa es evidente la preferencia de los diarios hacia el candidato del PRI, tanto en cantidad de notas dedicadas a él, como en el tono de la redacción. A pesar de que son titulares, se habló más a detalle de los objetivos de Roberto Loyola y se enfatizaron las críticas hacia el priísta por parte de su contrincante. La participación también es señalada a manera de inclusión.

"Voy a administrar el gobierno como las mujeres en casa": Loyola (5/05/15)

En este caso, se habla de un proyecto común que debe unir a todos los sectores. La nota refiere a las mujeres como un sector tradicionalmente marginado y a quien se convoca a participar por la importancia que refiere el candidato en tanto las destaca como “adminstradoras". Pero siempre quien lleva la voz es el actor político. En ningún momento se hace referencia a otros sectores.

En cuanto a la infraestructura, casi siempre se establece como parte de las "obligaciones" del estado. En algunas notas se ofrece esta como indispensable para el desarrollo social, como parte central del acceso a mejor calidad de vida. Todo esto bajo una lógica sobre la que se sustenta un discurso que afirma que desde el gobierno se debe trabajar en el desarrollo de infraestructura. Contrasta con la participación, en aquel discurso se incluía y se invitaba. En este discurso se establece que debe ser el gobierno quien provea. Aquí ya no hay inclusión hay "compromiso" por parte del estado.

"Búsqueda de mejora de condiciones de vida en las zonas rurales y urbanas a través de un diagnóstico de oportunidades, que permita la posibilidad de ofrecer proyectos productivos y de infraestructura" FDS (15/05/15)

"Siendo sensibles a las necesidades y problemas de la vida cotidiana, vamos a poder construir proyectos y soluciones reales" RLV (8/04/15)

En otro sentido, también sirve como argumento para señalar errores de "sensibilidad" del gobierno. 
"Las obras son suntuosas y de poco impacto social, lo cual ha caracterizado a los gobiernos panistas" FDS (15/04/15)

\section{c) Antagonismo}

Dependiendo del contexto que reseña la nota es el uso y la intencionalidad de los señalamientos. Aprovechando el foro, los actores utilizan las formas discursivas. No son mismos argumentos cuando el auditorio es favorable a cuando es una entrevista o cuando es un mitin de campaña. En cada caso el recurso y los argumentos son diferenciados. El periodista cuando escribe da cuenta de ello.

Aspiro a romper paradigmas: $R L V(27 / 05 / 15)$

En el caso de esta noticia, el evento es ante militantes de su propio partido, el actor aprovecha el foro para señalar que su gobierno será austero y lo plantea como un compromiso con la comunidad. En el mismo sentido el candidato a gobernador del PRI, en actos con militantes de su partido y mítines de campaña certificaba ante notario público sus promesas e incluso invitó a sus contrincantes, sostiene que eso fortalece el compromiso con la sociedad.

"Con la finalidad de que queden sustentadas para su cumplimiento, espero los demás candidatos lo hagan. Esto es un compromiso con la sociedad" RLV (27/05/15)

Cada pronunciamiento de los actores hace referencia a la posición desde la que hablan. La sociedad civil, expresa demandas, el político oferta soluciones. Existen actores como la iglesia católica, la universidad autónoma y los colegios de profesionistas que presentan posturas institucionales, con un discurso que pareciera sólo mostrar una visión parcial. Sin embargo, es constante que aparezcan como actores de la esfera pública con una participación activa. 
Uno de los argumentos que mantuvo constantes la iglesia católica fue: "la ciudadanía debe elegir, a partir del candidato que defienda los valores sociales y el respeto a la familia como base" o "no es posición de la iglesia promover un voto de castigo en el proceso electoral".

A pesar de parecer posicionamientos que no manifiestan preferencia alguna, sí tienen una tendencia clara que al lector le permiten construir una idea de hacia dónde se dirige el argumento de la jerarquía católica.

Cada actor muestra en el discurso su posición y desde donde habla. Queda de manifiesto que los lectores debemos tomar en cuenta este punto para entender la lógica de los argumentos y a qué se hace referencia con lo que se dice y los recursos que utiliza el actor para poner de manifiesto su perspectiva argumentativa.

\section{d) Posiciones y subjetividad política}

Este punto está relacionado con las prácticas a las cuales hace referencia el discurso. Los actores siempre aparecen en las notas con la posición que manejan. "candidato a..."; "presidente de..."; "secretario de..." Y bajo esta lógica utilizan todos los recursos discursivos para sostener sus argumentos.

Queda de manifiesto en las notas que esta posición es importante al momento de enfatizar la información. Es decir, no es lo mismo que la referencia se haga al candidato o al gobernador que a un miembro de la sociedad civil. En la noticia simplemente se reproduce lo que el actor político dice. Los periódicos intervienen en la ubicación de la nota en el cuerpo del periódico. Que, si bien dice mucho, el protagonista es el mensaje y quien lo dice.

En las notas revisadas es evidente que la lucha se da en la búsqueda por las posiciones de gobierno y encontrar en la población legitimidad a sus prácticas. Cada actor al momento de hacer referencia a sus perspectivas, utiliza argumentaciones para hacerse con el control del discurso. Sin embargo, llama la atención que son los argumentos semejantes. Solo que dichos de diferente manera; y ubicados en distintas páginas de los periódicos. 
Se considera que la prensa tiene muy claro a quien dar espacio y qué tipos de espacio dar a cada uno de los actores sociales. Esto es significativo porque si estamos hablando de proceso de alternancia electoral, la prensa debiera ser uno de los espacios más abiertos al debate, e incluso promoverlo. Sin embargo, a pesar del ímpetu de apertura de los años noventa e inicios del S. XXI, hoy en día es cada vez más difícil encontrar un espacio para debates más allá de los actores políticos. La negociación de los significados, en este sentido, queda claro que se da entre los actores políticos y la prensa. A partir de ellos se construye el discurso que se publica en la prensa.

\section{A manera de conclusión}

La ciudad de Querétaro es actualmente una de las ciudades con índices de crecimiento económico y de población más altos del país. A lo largo de distintos momentos históricos la sociedad queretana ha sufrido cambios, sin embargo, hay aspectos que al parecer han quedado inmóviles no obstante la serie de transformaciones que son visibles.

A nivel político el cambio más importante de los últimos años tuvo lugar en 1997 cuando un partido político de oposición ganó la alcaldía y el gobierno del estado. Este hecho consolidó una serie de cambios que ya venían de tiempo atrás con la llegada de organismos y personalidades a la vida política y económica tanto de la ciudad como del estado.

Pero aún quedan sectores en los cuales pareciera que estos cambios no hubieran tenido lugar. La prensa es uno de ellos. Con una ciudad en constante crecimiento económico y de población. La oferta periodística continuaba siendo la misma que en los años setenta. En la revisión hecha para el contexto sociohistórico se encuentra que la colusión entre la prensa y los poderes han pasado de acuerdos políticos a una subordinación a los intereses económicos. Esto pone de manifiesto que, a pesar de la apertura política, del proceso de democratización en el cual se encuentra el país, la prensa escrita continúa siendo un sector rezagado. 
Señala Lawson (2002) que existe una apertura paulatina que ha modificado el quehacer de los medios. A nivel nacional hay muestras de ello. En el caso de Querétaro también hay aspectos a destacar como el de las coberturas en las campañas electorales o los espacios partidos de oposición u organismos de la sociedad civil. Sin embargo, la forma en como es cubierta esta información y en cómo es reproducida, interpretada y publicada esta información permite observar que todavía queda camino por recorrer para hablar de una auténtica democratización en la prensa.

Cuando se llevó a cabo el análisis de la información se encontró que existen en ocasiones los mismos discursos en diferentes prensas. Es decir, notas informativas, casi iguales, relatando los mismos hechos, con casi los mismos énfasis. Cuando el lector se imaginaría encontrar lecturas diferenciadas de un mismo hecho, se lleva la sorpresa de que la información es la misma o casi la misma. En este sentido se puede sostener la idea de que la prensa en la ciudad de Querétaro reproduce el discurso de las fuentes, sirve de vehículo para la consolidación de un discurso dominante.

En lo que respecta a la forma de las notas, es prácticamente un relato de lo que señalan los actores. Lo que se puede leer es una reproducción discursiva. El periodista recoge lo que su fuente declara y lo expone en el formato de nota informativa. Los periódicos ofrecen a sus lectores una serie de notas construidas acríticamente. Dando más espacio a actores miembros de partidos políticos o de funcionarios gubernamentales y menos espacio para organizaciones civiles. Esta lectura que se ha adjetivado como acrítica, refiere a la falta de claridad en las líneas editoriales. En los cuatro periódicos no se puede establecer claramente a partir del discurso publicado en sus notas cuál es la línea editorial del periódico.

En este sentido, puede establecerse como una línea de debate el posicionamiento entorno a afirmar que los medios son el área más atrasada del proceso de democratización. Esto puede argumentarse señalando que, en la prensa de la ciudad de Querétaro, hay una discusión parcial respecto de los asuntos públicos. El discurso hegemónico, puesto en 
común por los poderes político y económico, es el que domina las páginas de los diarios. Si regresamos a los planteamientos de Dahl (1993), O’donell y Schmitter (1994), los medios, en particular la prensa queretana, no otorga los espacios, ni permite la apertura a negociar otras significaciones en sus páginas. La prensa refleja un pequeñísimo espacio de los debates que tienen lugar en la esfera pública.

Respecto a los temas seleccionados, son el pretexto, al menos durante procesos electorales, para hacer mención a los ofrecimientos de los candidatos que buscan el voto o buscar cargos de elección popular. Si bien, no se buscaba que hubiera señalamientos a nivel normativo. A partir del proceso de democratización se pensaría que la prensa destinara espacios a otros grupos de tal forma que se enriqueciera el debate público. Al estar inmersos dentro del inicio de un periodo electoral y durante el periodo electoral, la mayoría de las notas estaba enfocada hacia procesos de elecciones y ofertas de campaña.

Las noticias analizadas permiten conocer las articulaciones que se dan continuamente en las publicaciones de prensa. Los discursos que la prensa reproduce han permitido la consolidación de ciertas prácticas sociales y la conformación de poderes hegemónicos que, a partir del discurso logran sostenimiento y legitimación. En los años del PRI se legitimaban las prácticas políticas y ahora, a partir de los acuerdos económicos, se legitiman procesos de otro tipo. Pero lo que prevalece es el discurso dominante.

En México, se puede afirmar, ha habido una paulatina apertura en los medios. De hecho, en Querétaro, la oferta mediática pudo significar más espacios para otros actores sociales; sin embargo, poco a poco se han ido adecuando a reproducir las prácticas de producción informativa y de reproducción discursiva.

Finalmente, quienes siguen teniendo el control de debate, al menos en la prensa local de la ciudad de Querétaro, son los poderes tanto económico como político. Si Querétaro como se piensa es un botón de muestra del entorno nacional, se puede suponer que, en el proceso de democratización del país, sigue como agenda pendiente la apertura de espacios en los 
medios de difusión. Lo que se ve publicado son sólo reproducciones de un discurso producido por otros y no se lee en ningún espacio de prensa debates que se pudiera pensar enriquezcan a la esfera pública. El discurso de la prensa sigue en buena medida supeditado a negociaciones de poder. Lo cual se refleja en los textos periodísticos.

\section{Referencias Bibliográficas}

BERKOWITZ, Dan (ed.) (1997) Social meanings of news. A text reader. California, USA, SAGE.

CHALABY, Jean, K. (1998) Political communication in presidencial regimes in nonconsolidated democracies, en Gazette, Vol. 60 n. 5

CRICK, Bernard (2002) Democracy. A very short introduction. Oxford, UK: OUP

CURRAN, James (et. al) (2009) Media System, Public Knowledge and Democracy. European journal of communication Vol 24(1): 5-26. SAGE Publications.

DALH, Robert (1993) La democracia y sus críticos, Barcelona: Paidos (1989) La Poliarquía. Participación y oposición. Madrid: Tecnos.

DÍAZ, Ana (2002) Democratización y Alternancia. Encuesta de actitudes y orientaciones políticas de los ciudadanos en Querétaro, México: UAQ, UDG, IEQ

DUQUETTE, Michel (1999) Building new democracies. Toronto. University of Toronto Press

DEMERS, Francois \& Lavigne, A. (2007). La comunicación pública: una prioridad contemporánea de investigación. En Comunicación y Sociedad, No. 7, nueva época.

FAIRCLOUGH, Norman (1995) Media discourse. London, UK. Hodder Arnold.

FINLAYSON, James Gordon (2005) Habermas. A very short introduction. Oxford, UK: OUP

FREEDEN, Michael (1996) Ideologies and political theory: a conceptual aproach. Oxford, UK: OUP

GRAMSCI, Antonio (1971) Cuadernos de la Cárcel. México: ERA

CHAM Gerardo (2003) Teoría del Discurso (estrategias periodísticias) Guadalajara: UDG

HABERMAS, J. (1981) Historia y crítica de la opinión pública. La transformación estructural de la vida pública, México: Gustavo Gili

(1996) Between facts and norms. Boston: MIT 
(2006) Political Communication in Media Society: does Democracy still enjoy an epistemic dimension? The impact of normative theory on empirical research.

KRIPPENDORF, Klaus (1997) Metodología del análisis de Contenido. Teoría y Práctica. Barcelona. Paidos

LAWSON, Chappel H. (2002) Building the fourth estate. Democratization and the rise of a free press in México. California, US: University of Californa Press.

LEÓN y Ramírez, Juan Carlos (2004) La construcción de espacios púbicos en la democracia. México: UAEM

LÖFFELHOLZ, Martin and david Weaver (eds.) Global journalism research. Oxford, UK. Blackwell publishing.

MIDDELEBROOK, KEVIN (2004) Dilemmas of political change in Mexico. London, UK: Institute of Latin American Studies

MILLER David (2003) Political philosophy. A very short introduction. Oxford, UK: OUP

MINOGUE Kenneth (1995) Politics. A very short introduction. Oxford, UK: OUP

MORLINO, Leonardo (2005) Democracias y democratizaciones, México: Cepcom.

NEGRINE Ralph y James Stanyer (eds) (2007) The political communication reader. Oxford, UK: Routledge.

NORRIS, Pippa (2000) The news media and democracy \& a virtuos circle? en ibid. A virtuos circle. Political Communications in Post Industrial Societies Cambridge University Press. Cambridge

O'DONNELL, Guillermo, Schmitter, Philippe (1994) Transiciones desde un gobierno autoritario, Barcelona: Paidos

SINGER, Jane B. (2009) Separate Spaces. Discourse about the 2007 Scotish elections on a national newspaper web site. International Journal of press/politics V.14 n. 4 October 2009

THOMPSON, John B. (1993) Ideología y Cultura Moderna. México: UAM

(1998) Los media y la modernidad: una teoría de los medios de comunicación. Barcelona: Paidos.

TORFING, Jacob (1999) New Theories of Discourse: Laclau, Mouffe and Zizek. Oxford UK: Blackwell Publishers.

TUCHMAN, G (1972) Objectivity as strategic ritual: An examination of newsman notions of objectivity, American Journal of sociology Chicago: The University of Chicago Press 
(1978) Making news: A study in the construction of reality. New York: The Free press.

VAN DIJK, Teun (comp.) (2000) El discurso como estructura y proceso. Estudios sobre el discurso. Una introducción multidisciplinaria. Barcelona: Gedisa

VON SCHOMBERG, Rene and Kenneth Baynes (eds) (2002) Discourse and democracy. Seáis on Habermas's between facts and norms. New York, USA. SUNY.

WETHERELL, M, Taylor, S, Yates S. (2001) Discourse as data. A guide for analysis. London, UK. Sage publications; The Open University.

WHITEHEAD, Laurence (1997) The vexed issue of the meaning of "democracy" in Journal of political ideologies. Volume 2 number 2 june 1997. Oxford, UK: Carfax

(2003) Democratization Theory and Practice. Oxford, UK: OUP.

WOLTON Dominique. (1998). La comunicación política: construcción de un modelo. En Ferry et al, El nuevo espacio público. Barcelona: Gedisa.

\section{Forma de citar este artículo en bibliografías}

CORRAL, G. (2017): "Tratamiento informativo y cobertura electoral en Querétaro: Una mirada a la elección estatal de 2015”, en Revista PANGEA № 8, páginas 85 a 108. Tenerife: Red Académica Iberoamericana de Comunicación. Recuperado el de de 2 de: http://www.revistapangea.org 